\title{
PROBABILITY OF SEEING 〈001〉 CROSS-FRINGES IN A RANDOM CUBIC NANOCRYSTAL IMAGE
}

\author{
W. Qin and P. Fraundorf
}

Physics \& Astronomy Department and CME, University of Missouri-StL, St. Louis MO 63121

As a crystal gets smaller, diffraction spots stay visible for larger deviations from the Bragg condition. The upper limit of such deviations is connected to the threshold for getting lattice fringes in TEM images. ${ }^{1}$ This in turn allows one to quantify the probability of seeing cross lattice fringes along a certain zone axis. In this abstract we examine a simple semi-empirical model for the probability of detecting $\langle 001\rangle$ zone cross-fringes of a spherical crystal of cubic lattice.

The upper limit for the deviation of crystal orientation from the exact Bragg condition, without losing cross fringes down a given zone, is expressed as the maximum half-angle $\theta_{t}$ between the zone and the electron beam. The solid angle $\sigma$ subtended by a cone with this half-angle is proportional to the probability $\mathrm{p}_{\mathrm{x}}$ that a randomly-oriented crystal will show the cross-fringes associated with that zone. A schematic, illustrating the principle used to calculate the probability of seeing cross-fringes, is given in Figure 1. The resulting probability is

$$
\begin{aligned}
& p_{x} \cong \frac{n \sigma}{4 \pi}=\frac{n}{2}\left(1-\cos \theta_{t}\right), \text { where } \\
& \theta_{t}=\sin ^{-1} \frac{g_{l}^{2}-g_{d}^{2}+2 g_{\lambda} g_{t}}{\sqrt{2} g_{d} g_{\lambda}} .
\end{aligned}
$$

Here $\mathrm{n} \equiv$ zone multiplicity (e.g. $\mathrm{n}=6$ for $\langle 001\rangle$ zones), reciprocal lattice spacing $\mathrm{g}_{\mathrm{d}} \equiv 1 / \mathrm{d}$, Ewald sphere radius $\mathrm{g}_{\lambda} \equiv$ $1 / \lambda$, and the effective reciprocal-spot radius $g_{t} \equiv f / t$, where $d$ is the lattice spacing, $t$ is the diameter of the crystal, $\lambda$ is the electron wavelength, and visibility factor $\mathrm{f}$ is a parameter of order one that empirically adjusts for signal-to-noise in the method used to "visualize" fringes. Derivation of $p_{x}$ is given elsewhere. ${ }^{2}$

In determining $\mathrm{f}$, three HREM images of $\mathrm{Au} / \mathrm{Pd}$ evaporated onto a carbon film were analyzed. It is assumed that all the crystals are spherical and randomly oriented. The fractions of nanocrystals showing $\langle 001\rangle$ zone images and in two size ranges were used to estimate $\mathrm{f}$ from equation (1) by least squares fitting. ${ }^{2}$ The visibility factor $\mathrm{f}$ was thus estimated for this specimen to be $\cong 0.952$. Hence the effective radius of reciprocal spots for this specimen were nearly $1 / t$, as predicted.

A plot of the fit and inferred probabilities for seeing $\langle 001\rangle$ cross-fringes in $A u / P d$ crystals with spacing $d=2.04 \AA$ on thin carbon film, as a function of crystal size t, is shown in Figure 2. Note that the data points on the plot were used to determine $\mathrm{f}$ (and hence the height of the curve), and that the lateral bracket around each point denotes not a size uncertainty, but the range of sizes used in estimating the probability. As you can see, the probability of encountering cross-fringes improves from $10^{-4}$ to $10^{-2}$ as crystallite size decreases from $10 \mathrm{~nm}$ toward $1 \mathrm{~nm}$. Of course, this "reciprocal lattice broadening" will be accompanied by a slight increase in the range of spacings and interspot angles observed, due to projection foreshortening. ${ }^{2}$ For very small crystals, it may also be accompanied by curved fringes and deceptive moires of the projected potential. ${ }^{3}$

References:

1. W. Qin and P. Fraundorf, Lattice fringe visibility after tilt (this conference).

2. W. Qin and P. Fraundorf, Los Alamos' arXiv:cond-mat/0001139 (http://arXiv.org).

3. J.-O. Malm and M. A. O'Keefe, Ultramicroscopy 68 (1997) 13-23.

4. Acknowledgements: Dr. James and Dr. Shih at U. Missouri-Rolla for the WC film 


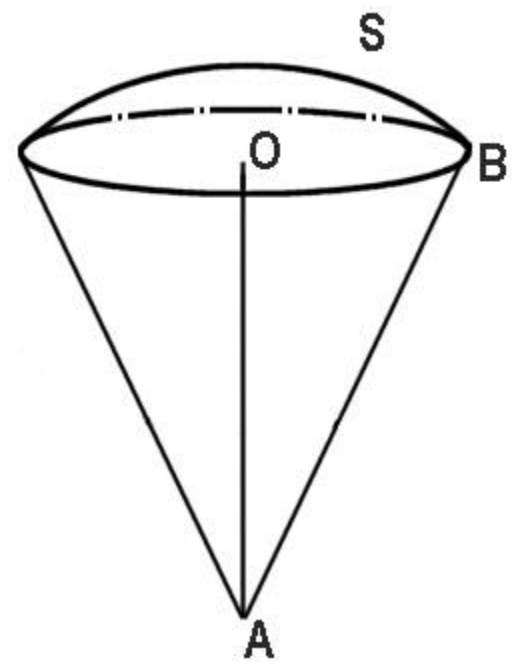

2

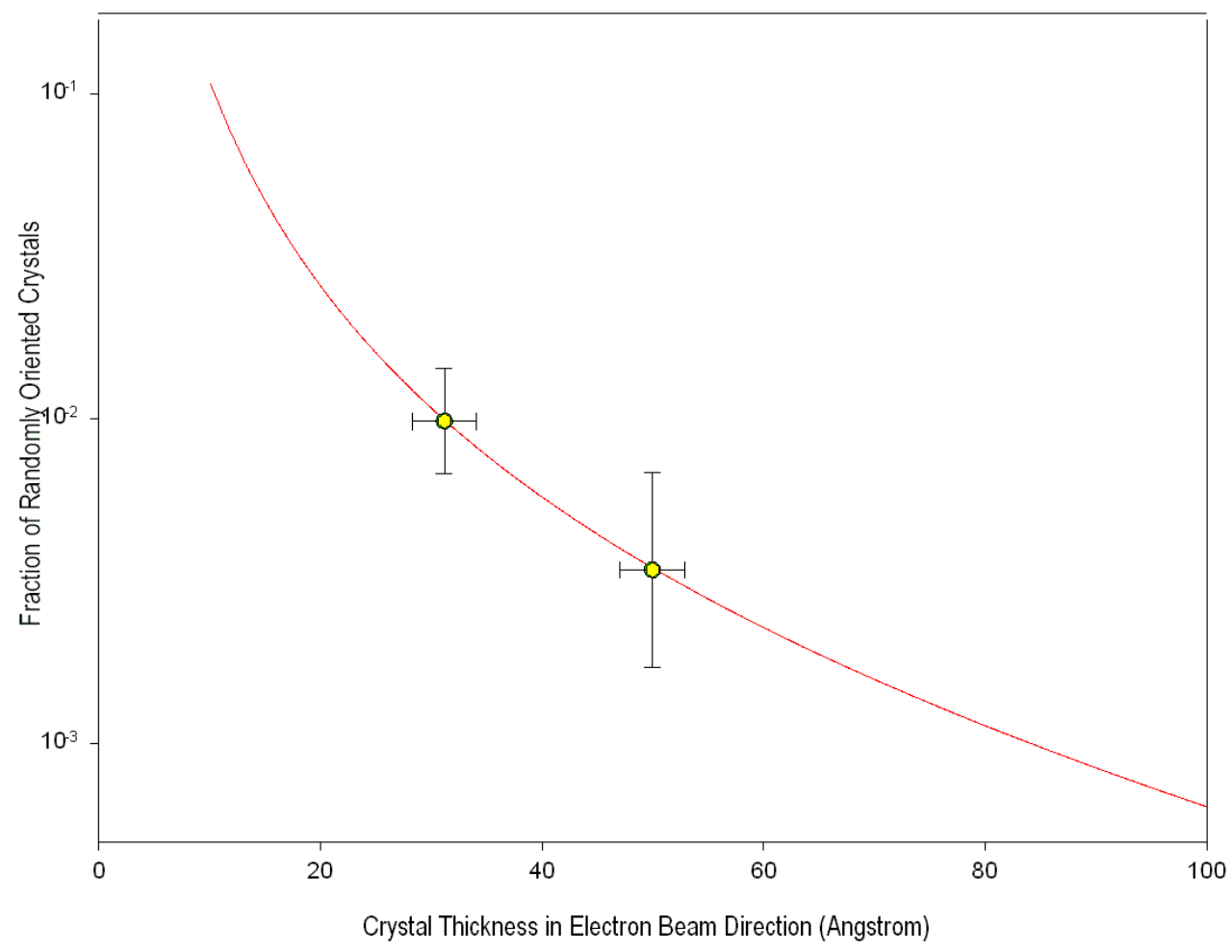

FIG. 1. Schematic illustrating the principle to calculate the probability of seeing cross-fringes along a zone axis of a randomly oriented crystal in HREM image. $\mathrm{OA}$ is parallel to the electron beam. $\angle \mathrm{OAB}=\theta_{\mathrm{t}}$ denotes a maximum angle between the zone axis and the electron beam without loosing contact of the Ewald sphere with the two reciprocal spots along the zone and hence without loosing cross-fringes in HREM images. The solid angles thus defined are proportional to the probability of encountering cross-fringes along the zone.

FIG. 2. The probability of getting cross fringes along a $\langle 001\rangle$ zone of Au/Pd particles as a function of crystal diameter. The experimental data of fractions of Au/Pd crystals showing cross fringes along $\langle 001\rangle$ zones are also shown. 